\title{
Patients with gunshot wounds to the torso differ in risk of mortality depending on treating hospital
}

\author{
Areg Grigorian $^{1}$ (D) Jeffry Nahmias ${ }^{1} \cdot$ Theresa Chin $^{1} \cdot$ Angela Allen $^{1} \cdot$ Eric Kuncir $^{1} \cdot$ Matthew Dolich $^{1} \cdot$ Victor Joe $^{1}$. \\ Michael Lekawa ${ }^{1}$
}

Received: 9 February 2019 / Accepted: 16 April 2019 / Published online: 22 April 2019

(C) Italian Society of Surgery (SIC) 2019

\begin{abstract}
Previous studies have had conflicting results when comparing risk of mortality in patients with gunshot wounds (GSWs) treated at Level-I and II trauma centers. However, the populations studied were restricted geographically. We hypothesized that patients presenting after a GSW to the torso at Level-I centers would have a shorter time to surgical intervention (exploratory laparotomy or thoracotomy) and a lower risk of mortality, compared to Level-IIs in a national database. The Trauma Quality Improvement Program (2010-2016) was queried for patients presenting to Level-I or II trauma center after a GSW to the torso. A multivariable logistic regression analysis was performed. From 17,965 patients with GSWs, 13,812 (76.8\%) were treated at Level-Is and $4153(23.2 \%)$ at Level-IIs. There was no difference in the injury severity score (ISS) $(p=0.55)$. The Level-I cohort had a higher rate of laparotomy $(38.9 \%$ vs. $36.5 \%, p<0.001)$ with a shorter median time to laparotomy (49 vs. $55 \mathrm{~min}, p<0.001)$ but no difference in rate $(p=0.14)$ and time to thoracotomy $(p=0.62)$. After adjusting for covariates, only patients undergoing thoracotomy $(\mathrm{OR}=0.66, \mathrm{CI}=0.47-0.95, p=0.02)$ or those undergoing non-operative management $(\mathrm{NOM})(\mathrm{OR}=0.85, \mathrm{CI}=0.74-0.98, p=0.03)$ at a Level-I center had lower risk for death, compared to Level-II. Patients with torso GSWs managed with thoracotomy or NOM at a Level-I center have a lower risk of mortality, compared to a Level-II. Future prospective studies examining variations in practice, resources available and surgeon experience to account for these differences are warranted.
\end{abstract}

Keywords Trauma Gunshot · Trauma center $\cdot$ Level-I

\section{Introduction}

Trauma is the leading cause of death for Americans under the age of 45 years and the fourth leading cause of death overall for all ages [1]. As one of the leading public health epidemics in the United States, the American College of Surgeons (ACS) has assumed the responsibility to verify trauma centers across the country to allow a consistent and optimal national trauma system [2]. This has resulted in the delivery of timely, definitive and organized care to injured persons with multiple studies demonstrating a significant reduction in mortality since its inception [3-5]. The ACS

Areg Grigorian

agrigori@uci.edu

1 Division of Trauma, Burns, Surgical Critical Care, and Acute Care Surgery, Department of Surgery, University of California, Irvine Medical Center, 333 The City Blvd West, Suite 1600, Orange, CA, USA 92868-3298 does not designate trauma centers but does verify the presence of the resources required to achieve a verification level as described in the Resources for Optimal Care of the Injured Patient [6]. The ACS currently offers five levels of verification (Level I-V) depending on the resources available, the processes in place and the annual case-volume of the hospital. Level-I and II centers are required to provide definitive care for all trauma patients. Notable exceptions at Level-I centers include immediate coverage by subspecialty services, as well as a commitment to teaching and research efforts to help direct new innovations in trauma care [7]. Hospital outcomes such as mortality, complications and quality-improvement metrics are not currently used in the verification process.

Differences in outcomes between Level-I and II centers have been mixed. Demetriades et al. reported improved survival in severely injured patients treated at a Level-I center compared to a Level-II [8]. This finding was reaffirmed in subsequent studies [9-11]. Haas et al. demonstrated that 
Level-II centers in close proximity to a Level-I center might perform at a lower than expected level suggesting differing roles depending on the local trauma system configuration [12]. Other studies report equal or improved outcomes at Level-II centers, compared to Level-I centers [13, 14]. Some authors propose total trauma center volume and indirectly, surgeon experience, and not trauma-level verification, is what drives improved outcomes and increased survival [15, 16]. A recent analysis of the Los Angeles County Trauma and Emergency Medical Information System found that treatment at a Level-II center was associated with an overall risk-adjusted reduced mortality compared to Level-I centers. However, this difference did not persist in a subset of penetrating trauma patients [17].

In the appropriate environment and setting, some civilian abdominal gunshot-wounds (GSWs) can be managed with nonoperative management (NOM), particularly tangential or when limited to the right upper quadrant $[18,19]$. Successful NOM depends on the treating hospital's facilities, resources, and surgeon or trainees' experience [20-22]. Thus, some variability exists, and the dogma of mandatory laparotomy has now ended. Most trauma surgeons would agree that there is less variability in the management of transthoracic GSWs. However, Bozdag et al. reported on the NOM of 13 select cases of thoracoabdominal gunshot injuries [23]. Clarifying outcomes depending on the treatment strategy provides the best prospect for improved survival. This can help ensure that optimal care is being delivered to patients with GSWs to the torso.

Many of the previous studies on differing outcomes between trauma centers was restricted geographically. The purpose of this study was to evaluate the differences among Level-I and II centers across the country in treating patients with GSWs to the torso. We hypothesized that patients presenting after a GSW to the torso at a Level-I center would have a shorter time to surgical intervention (exploratory laparotomy or thoracotomy), compared to a Level-II. We also hypothesized that patients with torso GSWs managed operatively at a Level-I center would have a lower risk of mortality.

\section{Methods}

This study was approved by the Institutional Review Board at the University of California, Irvine. A retrospective analysis of the Trauma Quality Improvement Program (TQIP) was performed over a 7-year period (2010-2016). International Classification of Diseases (ICD) Version-9 event codes were used to select for patients presenting after a gunshot wound. Patients without a documented treating hospital ACS designation level, and those with a Level-III designation or transferred from another hospital were excluded. TQIP does not enroll patients from hospitals with Level-IV or $\mathrm{V}$ trauma designation. Additionally, we excluded all patients with a severe, critical or maximum grade for the abbreviated injury scale (AIS) of the head, neck or extremities to select for patients with isolated torso GSWs. Two groups were compared: patients with torso GSWs treated at Level-I vs. Level-II trauma centers. The primary outcome was time to surgical intervention, defined by ICD- 9 procedure codes for laparotomy (54.11) and thoracotomy (34.02). The secondary outcome was all-cause mortality during the index hospitalization.

Demographic information collected included age, gender and comorbidities including cardiac disease, chronic renal failure, diabetes, chronic obstructive pulmonary disease and smoking. The injury profile consisted of AIS for the thorax and abdomen, hypotension on admission (systolic blood pressure $\leq 90 \mathrm{mmHg}$ ), as well as injuries to the lung, heart, spleen, liver, pancreas, small bowel, colon or rectum. These were defined by the appropriate ICD-9 diagnosis codes. Other outcomes measured included total hospital length of stay (LOS), intensive care unit (ICU) LOS, ventilator days, blood products transfused, thoracotomy for hemorrhage control, and in-hospital complications including venous thromboembolism, wound or deep space infection, acute kidney injury (AKI), acute respiratory distress syndrome and pneumonia.

Frequency statistics were performed for all variables. Continuous variables were analyzed with a Student's $t$ test or Mann-Whitney- $U$ test, while categorical variables were analyzed with the Chi-squared test. A review of the literature was performed to identify independent predictors of mortality in trauma [24-26]. The magnitude of the association between predictor variables and mortality was first measured using a univariable logistic regression model. Covariates were then controlled for in a hierarchical multivariable logistic regression model and the adjusted risk for mortality was reported with an odds ratio (OR) and 95\% confidence intervals (CI). All $p$ values were two-sided, with a statistical significance level of $<0.05$. All analyses were performed with IBM SPSS Statistics for Windows (Version 24, IBM Corp., Armonk, NY, USA).

\section{Results}

\section{Demographics and primary outcomes of torso GSWs}

From 17,965 patients with torso GSWs, 13,812 (76.8\%) were treated at a Level-I and $4153(23.2 \%)$ at a Level-II center. Compared to those at a Level-II, the Level-I cohort had a higher rate of smokers $(22.8 \%$ vs. $19.7 \%, p<0.001)$. There was no difference in other patient demographics as well as the injury profile except for a higher rate of pancreatic 
injuries in patients treated at a Level-I center (4.8\% vs. $3.8 \%, p=0.01)$. The median ISS was similar $(14, p=0.08)$. (Table 1). The Level-I group had a higher rate of laparotomy $(38.9 \%$ vs. $36.5 \%, p<0.001)$ with a shorter median time to laparotomy (49 vs. $55 \mathrm{~min}, p<0.001$ ) but no difference in the rate (Level-I 7.6\% vs. Level-II 6.9\%, $p=0.14$ ) or time to thoracotomy (Level-I 46 vs. Level-II $41 \mathrm{~min}, p=0.62$ ).

\section{Mortality in trauma patients with torso GSWs}

In a univariable logistic regression model, patients treated at a Level-I center had a lower risk of mortality (OR 0.89, CI $0.81-0.98, p=0.02$ ), compared to those treated at a LevelII. This finding persisted after adjusting for covariates (OR 0.85 , CI $0.74-0.97, p=0.02$ ). The strongest independent risk factor was hypotension on admission (OR 8.11, CI 7.19-9.14, $p<0.001$ ) (Table 2). Patients at a Level-I center managed with laparotomy $(11.5 \%$ vs. $13.8 \%, p=0.02)$ or thoracotomy $(50.8 \%$ vs. $61.5 \%, p=0.01)$ and those that underwent non-operative management $(12.8 \%$ vs. $14.0 \%$, $p=0.04$ ) all had a lower rate of mortality. On multivariable logistic regression analysis, only patients that underwent thoracotomy (OR 0.66 , CI $0.47-0.95, p=0.02$ ) or NOM
$(\mathrm{OR}=0.85, \mathrm{CI}=0.74-0.98, p=0.03)$ at a Level-I center had lower risk for death, compared to those treated at a LevelII. The risk of mortality in patients with torso GSWs managed with a laparotomy at Level-I and II centers was similar $(p=0.08)$ (Table 3). We also looked at patients older than 65 years, and in this patient group there was no statistical difference between Level-I and II centers with regard to mortality rate (LI 30.7\% vs. LII 40.0\%, $p=0.08$ ) and adjusted mortality risk (OR $0.62,0.35-1.09, p=0.10$ ). In patients younger than 65 years of age, there was no statistical difference between Level-I and II centers with regard to mortality rate (LI $13.6 \%$ vs. LII $14.7 \%, p=0.06$ ) but there was a decreased adjusted mortality risk (OR 0.86, 0.75-0.99, $p=0.04$ ).

\section{Other clinical outcomes in torso GSWs}

Compared to patients at a Level-II, those treated at a Level-I center had a longer median ICU LOS ( 4 vs. 3 days, $p=0.01$ ) but no difference in total hospital LOS or ventilator days $(p>0.05)$. The Level-I cohort had a higher rate of AKI $(2.4 \%$ vs. $1.6 \%, p=0.01)$ and pneumonia (5.5\% vs. $4.3 \%$, $p=0.01)$ (Table 4).
Table 1 Demographics of adult trauma patients presenting after a torso gunshot-wound

\begin{tabular}{|c|c|c|c|}
\hline \multirow[t]{2}{*}{ Characteristic } & \multicolumn{3}{|l|}{ Gunshot wounds } \\
\hline & ACS Level-I $(n=13812)$ & ACS Level-II $(n=4153)$ & $p$ value \\
\hline Age, year, median (IQR) & $27.0(21,37)$ & $27.0(21,37)$ & 0.15 \\
\hline Male, $n(\%)$ & $12311(89.1 \%)$ & $3658(88.1 \%)$ & 0.08 \\
\hline ISS, median (IQR) & $14.0(9,24)$ & $14.0(9,22)$ & 0.55 \\
\hline $\begin{array}{l}\text { Lowest SBP within } 24 \mathrm{~h} \text {, } \\
\text { median (IQR) }\end{array}$ & $84.0(56,108)$ & $85.0(60,107)$ & 0.57 \\
\hline \multicolumn{4}{|l|}{ Comorbidities, $n(\%)$} \\
\hline Congestive heart failure & $39(0.3 \%)$ & $9(0.2 \%)$ & 0.47 \\
\hline End-stage renal disease & $14(0.1 \%)$ & $7(0.2 \%)$ & 0.27 \\
\hline Smoker & $3152(22.8 \%)$ & $773(19.7 \%)$ & $<0.001$ \\
\hline Diabetes & $351(2.5 \%)$ & $98(2.4 \%)$ & 0.51 \\
\hline Hypertension & $1010(7.3 \%)$ & $306(7.4 \%)$ & 0.90 \\
\hline COPD & $567(4.1 \%)$ & $154(3.7 \%)$ & 0.25 \\
\hline \multicolumn{4}{|l|}{ AIS (grade $>3), n(\%)$} \\
\hline Thorax & $2246(16.3 \%)$ & $631(15.2 \%)$ & 0.10 \\
\hline Abdomen & $2890(20.9 \%)$ & $857(20.6 \%)$ & 0.69 \\
\hline \multicolumn{4}{|l|}{ Injuries, $n(\%)$} \\
\hline Lung & $7784(56.4 \%)$ & $2336(56.4 \%)$ & 0.90 \\
\hline Heart & $652(4.7 \%)$ & $215(5.2 \%)$ & 0.23 \\
\hline Liver & $3362(24.3 \%)$ & $1013(24.4 \%)$ & 0.95 \\
\hline Spleen & $1270(9.2 \%)$ & $353(8.5 \%)$ & 0.17 \\
\hline Small bowel & $3122(22.6 \%)$ & $943(22.7 \%)$ & 0.89 \\
\hline Colorectal & $2910(21.1 \%)$ & $912(22.0 \%)$ & 0.22 \\
\hline Pancreas & $659(4.8 \%)$ & $158(3.8 \%)$ & 0.01 \\
\hline
\end{tabular}

ACS American College of Surgeons, ISS injury severity score, IQR interquartile range, SBP systolic blood pressure, $A I S$ abbreviated injury scale, $C O P D$ chronic obstructive pulmonary disease 
Table 2 Multivariable logistic regression analysis for risk of mortality after a torso gunshot-wound

\begin{tabular}{lllc}
\hline Risk factor & OR & CI & $p$ value \\
\hline ACS Level-I vs II & 0.85 & $0.74-0.97$ & 0.02 \\
Age & 1.02 & $1.01-1.02$ & $<0.001$ \\
Hypotensive on admission & 8.11 & $7.19-9.14$ & $<0.001$ \\
ISS $\geq 25$ & 2.48 & $2.16-2.86$ & $<0.001$ \\
PRBC transfusion $\geq 6$ units within $4 \mathrm{~h}$ & 2.52 & $2.06-3.09$ & $<0.001$ \\
Severe AIS-thorax (grade $>3)$ & 2.72 & $2.34-3.16$ & $<0.001$ \\
Severe AIS-abdomen $($ grade $>3)$ & 2.37 & $2.04-2.76$ & $<0.001$ \\
Smoker & 0.12 & $0.09-0.16$ & $<0.001$ \\
Spleen injury & 0.46 & $0.37-0.56$ & $<0.001$ \\
Colorectal injury & 1.01 & $0.86-1.17$ & 0.95 \\
Pancreas injury & 2.15 & $1.70-2.71$ & $<0.001$ \\
Heart injury & 4.46 & $3.56-5.58$ & $<0.001$ \\
Acute kidney injury & 3.44 & $2.47-4.79$ & $<0.001$ \\
Superficial infection & 0.20 & $0.08-0.49$ & 0.001 \\
Organ space infection & 0.06 & $0.03-0.14$ & $<0.001$ \\
Deep vein thrombosis & 0.20 & $0.12-0.32$ & $<0.001$ \\
Pulmonary embolus & 0.17 & $0.07-0.37$ & $<0.001$ \\
Pneumonia/VAP & 0.39 & $0.30-0.51$ & $<0.001$ \\
\hline
\end{tabular}

ACS American College of Surgeons, ISS injury severity score, $P R B C$ packed red blood cell transfusion, VAP ventilator associated pneumonia, AIS abbreviated injury scale

\section{Discussion}

This retrospective analysis using 7 years of national data demonstrates the mortality rate of patients with GSWs to the torso managed either operatively or non-operatively to be lower when treated at Level-I vs. Level-II trauma centers. However, after adjusting for known predictors of mortality in trauma, only patients managed with thoracotomy or non-operatively at Level-I centers had a reduced risk of death, compared to Level-IIs. The median time to laparotomy was shorter at a Level-I but there was no difference in the time to thoracotomy between the two trauma centers.

Previous authors have demonstrated improved survival in trauma patients treated at Level-I centers. However, most of the previous literature has focused on select trauma populations $[27,28]$. Demetriades et al. reported on trauma patient $\geq 14$ years old with ISS $>15$ and found Level-I centers to have considerably better survival outcomes compared to Level-II centers [8]. Cudnik et al. performed a multicenter, retrospective analysis of Level-I and II trauma centers in the state of Ohio and found those treated at a Level-I center to have improved survival and were more likely to be discharged home [10]. A 10-year analysis of the Pennsylvania Trauma Outcomes Study registry also found a lower risk of mortality at Level-I vs. Level-II centers [29]. Similar results have been reported in head-injured patients $[11,28]$. Norwood et al. studied differences between Level-I and II trauma centers in patients with major torso vascular injuries. They found a significant reduction in those with penetrating thoracic trauma treated at Level-I centers ( $30 \%$ vs. $73 \%, p<0.001$ ) but no difference in those with major abdominal vascular injuries [30]. More recently, an analysis using the Los Angeles Country Trauma and Emergency Medical Information System found no differences in mortality between Level-I and II centers treating penetrating trauma. However, they did not differentiate outcome differences between those with stab-wounds or GSWs or between body compartments injured. Our study is the first to demonstrate up to a $15 \%$ reduced risk of death when patients with torso GSWs are treated at a Level-I center. Level-I centers are largely at academic institutions which often have surgical residents. Centers with a general surgery training program have been demonstrated to have higher observed survival than predicted models, compared to centers without trainees [31]. Additionally, Level-I centers with trainees engaged in critical care or trauma research may lead to modified institutional practices and protocols leading to improved outcomes [32-34]. Institutions involved in research may also be better able to recruit experienced and qualified faculty and intensivists which may be in-house and immediately available.

Not all patients with abdominal GSWs are managed operatively. Over the past 2 decades, there has been a concerted effort to better identify patients that would benefit from non-operative management. Factors favoring successful
Table 3 Adjusted* analysis for risk of mortality after a torso gunshot-wound at Level-I vs. II

\begin{tabular}{llllll}
\hline Risk factor & Mortality, $n(\%)$ & $p$ value & OR & CI & $p$ value \\
\hline All patients & $14.0 \%$ vs. $15.4 \%$ & 0.02 & 0.85 & $0.74-0.97$ & 0.02 \\
Managed with exploratory laparotomy & $11.5 \%$ vs. $13.8 \%$ & 0.02 & 0.83 & $0.67-1.02$ & 0.08 \\
Managed with exploratory thoracotomy & $50.8 \%$ vs. $61.5 \%$ & 0.01 & 0.66 & $0.47-0.95$ & 0.02 \\
Managed non-operatively & $12.8 \%$ vs. $14.0 \%$ & 0.04 & 0.85 & $0.74-0.98$ & 0.03 \\
\hline
\end{tabular}

*Each model controlled for: age, hypotension on admission, injury severity score $\geq 25$, packed red blood cell transfusion $\geq 6$ units within $4 \mathrm{~h}$, spleen, heart, pancreas or colorectal injury, severe abbreviated injury scale-thorax and abdomen (grade $>3$ ), smoker, deep vein thrombosis, pulmonary embolus, pneumonia, acute kidney injury, superficial infection or organ space infection 
Table 4 Clinical outcomes in adult trauma patients presenting after a torso gunshot wound

\begin{tabular}{|c|c|c|c|}
\hline \multirow[t]{2}{*}{ Outcome } & \multicolumn{3}{|l|}{ Gunshot wounds } \\
\hline & ACS Level-I $(n=13812)$ & ACS Level-II $(n=4153)$ & $p$ value \\
\hline LOS, days, median (IQR) & $7.0(4,13)$ & $7.0(4,12)$ & 0.11 \\
\hline ICU, days, median (IQR) & $4.0(2,8)$ & $3.0(2,6)$ & 0.01 \\
\hline Ventilator, days, median (IQR) & $2.0(1,6)$ & $2.0(1,5)$ & 0.70 \\
\hline PRBC units transfused within $4 \mathrm{~h}$, median (IQR) & $4.0(2,9)$ & $4.0(2,9)$ & 0.49 \\
\hline Exploratory laparotomy, $n(\%)$ & $5367(38.9 \%)$ & $1514(36.5 \%)$ & 0.01 \\
\hline Minutes to laparotomy, median (IQR) & $49(31,70)$ & $55(35,82)$ & $<0.001$ \\
\hline Exploratory thoracotomy, $n(\%)$ & $1053(7.6 \%)$ & $288(6.9 \%)$ & 0.14 \\
\hline Minutes to thoracotomy, median (IQR) & $46(18,113)$ & $41(14,75)$ & 0.62 \\
\hline \multicolumn{4}{|l|}{ Complications, $n(\%)$} \\
\hline Acute kidney injury & $334(2.4 \%)$ & $65(1.6 \%)$ & 0.01 \\
\hline Acute respiratory distress syndrome & $321(2.3 \%)$ & $90(2.2 \%)$ & 0.55 \\
\hline Deep vein thrombosis & $398(2.9 \%)$ & $101(2.4 \%)$ & 0.12 \\
\hline Pulmonary embolism & $183(1.3 \%)$ & $39(0.9 \%)$ & 0.05 \\
\hline Superficial infection & $225(1.6 \%)$ & $46(1.1 \%)$ & 0.02 \\
\hline Organ space infection & $378(2.7 \%)$ & $86(2.1 \%)$ & 0.02 \\
\hline Pneumonia & $763(5.5 \%)$ & $179(4.3 \%)$ & 0.01 \\
\hline
\end{tabular}

ACS American College of Surgeons, LOS length of stay, IQR interquartile range, ICU intensive care unit, $P R B C$ packed red blood cells

non-operative management include patients that are hemodynamically stable and without signs of diffuse peritonitis or evisceration. In patients selected for non-operative management, the most important principal is the abdominal exam performed multiple times by qualified surgeons or trainees. In a systematic review and meta-analysis, the pooled estimate of the risk of failure for non-operative management in abdominal GSWs was 7\% [22]. In our study, patients with torso GSWs undergoing non-operative management at Level-I centers had a lower risk of mortality, compared to those treated at Level-II centers. This may be due to patients receiving serial abdominal examinations by the same person, often a surgical resident, which has been demonstrated to be associated with less patients failing non-operative management, compared to other hospital personnel [22].

Time to intervention and definitive care is vital for patients with high-velocity injuries. Our study found that Level-I centers performing thoracotomies had more than a $30 \%$ reduced risk of mortality, compared to Level-II centers. Massive hemorrhage can occur in more than $90 \%$ of transthoracic penetrating trauma and is associated with high mortality [35]. Our findings support this as hemodynamic instability as a result of hemorrhagic shock was the most significant predictor of mortality in torso GSWs. These patients often require a massive transfusion protocol. Hamidi et al. noted that patients receiving massive transfusion at Level-I centers have up to a $25 \%$ reduced risk of mortality, compared to patients receiving massive transfusion at Level-II centers [36]. Other possible explanations include earlier involvement of in-house surgeons, multiple surgeon and resident availability at Level-I centers, and increased trauma surgeon's experience with GSW management.

Locoregional hospital features may be more appropriate predictors of morbidity and mortality in trauma patients. Additionally, there appears to be variation in clinical outcomes even among designated Level-I centers suggesting a quality chasm in trauma care. In patients with mild injuries, survival may differ in up to $11 \%$ of designated Level-I centers while in patients with severe injuries, $21 \%$ of centers had outcome disparities [37]. Other reports have suggested the volume of patients treated has a direct impact on clinical outcome [31]. Minei et al. found that as the patient volume increased at a trauma center, there was an associated increased survival, more ventilator-free days and less severe organ dysfunction [15]. Furthermore, annual trauma volume may be a surrogate for surgeon experience. Using a statewide trauma registry, Konvolinka et al. were able to demonstrate normalized survival for the institution when trauma surgeons treated a minimum number of adults with blunt trauma annually. However, in patients with penetrating trauma, surgeon experience did not contribute significantly to mortality [16]. Trauma volume may be less important in patients with minor injuries. Nathens et al. found highvolume trauma centers to have improved mortality when treating patients presenting with hemodynamic instability after penetrating abdominal injuries but no difference in those with normal vitals [38].

This study is a retrospective database analysis and inherently limited by reporting and coding errors. The TQIP database only offers data from the index hospitalization and so 
long-term results are lacking from this study. Other important variables that would be pertinent to our study that are missing in the database include surgeon experience, trauma center volume, subspecialty services available and adjuncts used in the non-operative management of patients (e.g., computed-tomography or ultrasound). There may also be a selection bias and an effect from unknown confounding variables on the risk of mortality between Level-I and II trauma centers.

\section{Conclusion}

Patients with GSWs managed with thoracotomy or nonoperatively at a Level-I center have a lower risk of mortality, compared to a Level-II. This may illustrate an opportunity to improve outcomes through trauma system structure and emergency-medical-service triage of patients with torso GSWs. Future prospective studies examining variations in practice, resources available and surgeon experience to account for these differences are warranted and to determine optimal pre-hospital trauma designation for this population.

Acknowledgements This research did not receive any specific grant from funding agencies in the public, commercial, or not-for-profit sectors.

Funding No funding was received for this work.

\section{Compliance with ethical standards}

Conflict of interest The authors declare that they have no conflicts of interest.

Research involving human and animal participants This research involved humans. However, since this retrospective study was performed using a national database with the identified patients, risk to participants is minimal.

Informed consent There is no consent required.

\section{References}

1. National Vital Statistics System. National Center for Health Statistics, CDC (2004)

2. Nathens AB, Jurkovich GJ, Cummings P, Rivara FP, Maier RV (2000) The effect of organized systems of trauma care on motor vehicle crash mortality. JAMA 283(15):1990-1994

3. Barquist E, Pizzutiello M, Tian L, Cox C, Bessey PQ (2000) Effect of trauma system maturation on mortality rates in patients with blunt injuries in the Finger Lakes Region of New York State. J Trauma Acute Care Surg 49(1):63-70

4. Papa L, Langland-Orban B, Kallenborn C et al (2006) Assessing effectiveness of a mature trauma system: association of trauma center presence with lower injury mortality rate. J Trauma Acute Care Surg 61(2):261-267
5. Durkin M, McElroy J, Guan H, Bigelow W, Brazelton T (2005) Geographic analysis of traffic injury in Wisconsin: impact on case fatality of distance to level I/II trauma care. Wis Med J 104(2):26-31

6. Trauma ACoSCo (1990) Resources for optimal care of the injured patient. Am College of Surgeons

7. American College of Surgeons. New Criteria Quick Reference Guide. https://wwwfacsorg//media/files/quality\%20programs/ trauma/vrc\%20resources/1_chapter_23\%20new\%20criteria\%20 reference\%20guide\%20v1ashx11. Accessed December 10, 2016

8. Demetriades D, Martin M, Salim A et al (2006) Relationship between American College of Surgeons trauma center designation and mortality in patients with severe trauma (injury severity score > 15). J Am Coll Surg 202(2):212-215

9. Glance LG, Osler TM, Mukamel DB, Dick AW (2012) Impact of trauma center designation on outcomes: is there a difference between Level I and Level II trauma centers? J Am Coll Surg 215(3):372-378. https://doi.org/10.1016/j.jamcollsur g.2012.03.018

10. Cudnik MT, Newgard CD, Sayre MR, Steinberg SM (2009) Level I versus Level II trauma centers: an outcomes-based assessment. J Trauma 66(5):1321-1326. https://doi.org/10.1097/ TA.0b013e3181929e2b

11. McConnell KJ, Newgard CD, Mullins RJ, Arthur M, Hedges JR (2005) Mortality benefit of transfer to level I versus level II trauma centers for head-injured patients. Health Serv Res 40(2):435-458

12. Haas B, Gomez D, Neal M, Hoeft C, Ahmed N, Nathens AB (2011) Good neighbors? The effect of a level 1 trauma center on the performance of nearby level 2 trauma centers. Ann Surg 253(5):992-995. https://doi.org/10.1097/SLA.0b013e318212234 6

13. Brown JB, Watson GA, Forsythe RM et al (2013) American College of Surgeons trauma center verification versus state designation: are Level II centers slipping through the cracks? J Trauma Acute Care Surg 75(1):44

14. Dufresne P, Moore L, Tardif P-A et al (2017) Impact of trauma centre designation level on outcomes following hemorrhagic shock: a multicentre cohort study. Can J Surg 60(1):45

15. Minei JP, Fabian TC, Guffey DM et al (2014) Increased trauma center volume is associated with improved survival after severe injury: results of a Resuscitation Outcomes Consortium study. Ann Surg 260(3):456-464. https://doi.org/10.1097/sla.00000 00000000873 (discussion 464-455)

16. Konvolinka CW, Copes WS, Sacco WJ (1995) Institution and persurgeon volume versus survival outcome in Pennsylvania's trauma centers. Am J Surg 170(4):333-340

17. Kaji AH, Bosson N, Gausche-Hill M et al (2017) Patient outcomes at urban and suburban level I versus level II trauma centers. Ann Emerg Med 70(2):161-168. https://doi.org/10.1016/j.annemergme d.2017.01.040

18. Demetriades D, Velmahos G, Cornwell E et al (1997) Selective nonoperative management of gunshot wounds of the anterior abdomen. Arch Surg (Chicago, IL: 1960) 132(2):178-183

19. Velmahos GC, Demetriades D, Toutouzas KG et al (2001) Selective nonoperative management in 1,856 patients with abdominal gunshot wounds: should routine laparotomy still be the standard of care? Ann Surg 234(3):395-402 (discussion 402-393)

20. Cowey A, Mitchell P, Gregory J, Maclennan I, Pearson R (2004) A review of 187 gunshot wound admissions to a teaching hospital over a 54-month period: training and service implications. Ann R Coll Surg Engl 86(2):104-107. https://doi.org/10.1308/00358 8404322827482

21. Velmahos GC, Demetriades D, Foianini E et al (1997) A selective approach to the management of gunshot wounds to the back. Am J Surg 174(3):342-346 
22. Al Rawahi AN, Al Hinai FA, Boyd JM et al (2018) Outcomes of selective nonoperative management of civilian abdominal gunshot wounds: a systematic review and meta-analysis. World J Emerg Surg: WJES 13:55. https://doi.org/10.1186/s13017-018-0215-0

23. Bozdag Z, Turkoglu A, Gumus M et al (2015) Non-operative management of thoracoabdominal gunshot injury: thirteen unusual cases. J Curr Surg 5(4):199-203

24. Calland JF, Xin W, Stukenborg GJ (2013) Effects of leading mortality risk factors among trauma patients vary by age. J Trauma Acute Care Surg 75(3):501-505. https://doi.org/10.1097/ TA.0b013e31829bb75c

25. Emircan S, Ozguc H, Akkose Aydin S, Ozdemir F, Koksal O, Bulut M (2011) Factors affecting mortality in patients with thorax trauma. Turkish J Trauma Emerg Surg: TJTES 17(4):329-333

26. Goris R, Draaisma J (1982) Causes of death after blunt trauma. J Trauma Acute Care Surg 22(2):141-146

27. Demetriades D, Martin M, Salim A, Rhee P, Brown C, Chan L (2005) The effect of trauma center designation and trauma volume on outcome in specific severe injuries. Ann Surg 242(4):512-517 (discussion 517-519)

28. DuBose JJ, Browder T, Inaba K, Teixeira PG, Chan LS, Demetriades D (2008) Effect of trauma center designation on outcome in patients with severe traumatic brain injury. Arch Surg (Chicago, IL: 1960) 143(12):1213-1217. https://doi.org/10.1001/archs urg.143.12.1213 (discussion 1217)

29. Glance LG, Li Y, Osler TM, Mukamel DB, Dick AW (2014) Impact of obesity on mortality and complications in trauma patients. Ann Surg 259(3):576-581. https://doi.org/10.1097/ sla.0000000000000330

30. Norwood S, Cook AD, Berne JD (2011) Level I verification is associated with a decreased mortality rate after major torso vascular injuries. Am Surg 77(1):32-37

31. Pasquale MD, Peitzman AB, Bednarski J, Wasser TE (2001) Outcome analysis of Pennsylvania trauma centers: factors predictive of nonsurvival in seriously injured patients. J Trauma 50(3):465472 (discussion 473-464)
32. Black SR, Sathy AK, Jo C, Wiley MR, Minei JP, Starr AJ (2016) Improved survival after pelvic fracture: 13-year experience at a single trauma center using a multidisciplinary institutional protocol. J Orthop Trauma 30(1):22-28. https://doi.org/10.1097/ bot.0000000000000443

33. Nickele CM, Kamps TK, Medow JE (2013) Safety of a DVT chemoprophylaxis protocol following traumatic brain injury: a single center quality improvement initiative. Neurocrit Care 18(2):184-192. https://doi.org/10.1007/s12028-012-9786-x

34. Moydien MR, Oodit R, Chowdhury S, Edu S, Nicol AJ, Navsaria PH (2016) Enhanced recovery after surgery (ERAS) in penetrating abdominal trauma: a prospective single-center pilot study. S Afr J Surg 54(4):7-10

35. Givergis R, Munnangi S, Fayaz MFK, Boutin A, Zapata LC, Angus LG (2018) Evaluation of massive transfusion protocol practices by type of trauma at a level I trauma center. Chin J Traumatol 21(5):261-266. https://doi.org/10.1016/j.cjtee.2018.01.005

36. Hamidi M, Zeeshan M, Kulvatunyou N et al (2019) Outcomes after massive transfusion in trauma patients: variability among trauma centers. J Surg Res 234:110-115. https://doi.org/10.1016/j. jss.2018.09.018

37. Shafi S, Friese R, Gentilello LM (2008) Moving beyond personnel and process: a case for incorporating outcome measures in the trauma center designation process. Arch Surg (Chicago, IL: 1960) 143(2):115-119. https://doi.org/10.1001/archsurg.2007.29 (discussion 120)

38. Nathens AB, Jurkovich GJ, Maier RV et al (2001) Relationship between trauma center volume and outcomes. JAMA 285(9):1164-1171

Publisher's Note Springer Nature remains neutral with regard to jurisdictional claims in published maps and institutional affiliations. 